1144

\section{OUTCOMES OF NEONATAL EXCHANGE TRANSFUSION - 11 YEAR EXPERIENCE AT A TERTIARY CENTRE IN THE UK}

P. Chandra, A.K. Ewer, S.V. Rasiah

Neonatolgy, Birmingham Women's Foundation NHS Trust, Birmingham, UK

Background: There are limited data on the outcome of babies following exchange transfusions(ExT).

Aims: To review and define the postnatal outcomes in babies who received ExT.

Methods: All babies receiving ExT between 01/01/96 to $31 / 12 / 06$ were identified using neonatal database. Data were extracted from the case notes.

Results: 140 ExTs were performed in 108 neonates. 106 (98\%) babies survived to discharge. Indications for ExT included jaundice in 83/140 (59\%), anaemia in 56/140 (40\%), and DIC in 1/140. 131/140 (94\%) were double and 9/140 (6\%) were single volume ExTs. $78 \%$ of ExTs were performed using UAC remaining using UVC or peripheral arteries. In 124/140 (89\%) there were no procedural complications. There were mild complications in $11 / 140(8 \%)$ requiring either no or minimal intervention. ExT was abandoned in 5/140 $(4 \%)$ due to difficulty in sampling or aspiration of blood. Two babies died i) DIC ii) hydrops. One baby developed NEC. Although there were significant falls in the post ExT platelet count and calcium, only 3 required platelet transfusion and none required calcium correction. Of the 106 babies surviving to discharge, 94 were followed up to at least one year. One died at 5 months (SIDS) and 11 were transferred elsewhere. 91/94 (97\%) had normal hearing, vision and development. 3 babies had sensori-neural deafness and speech delay, one baby born at 33 weeks developed spastic quadriplegia and global developmental delay.

Conclusion: ExT has good safety profile and effective treatment outcome. In our experience, there were no significant sequelae attributable to the procedure.
1145

\section{THE STRUCTURE AND INCIDENCE OF MORBIDITY IN NEWBORNS AFTER ASSISTED FERTILIZATION IVF}

\author{
O.V. Laban', S.D. Obradovic ${ }^{2}$, P. Sazdanovic ${ }^{1}$, \\ L. Bekcic ${ }^{1}$, A. Busarac ${ }^{1}$ \\ ${ }^{1}$ Gynecology-Obstetrics Clinic, ${ }^{2} P e d i a t r i c ~ C l i n i c$, \\ Clinical Center Kragujevac, Kragujevac, Serbia
}

Assisted fertilization (IVF-ET) of 1978. becomes a standard method in medical practice that. About $20 \%$ of married couples suffer from infertility in Serbia and every sixth couple in Kragujevac. IVFET is the method of choice for about $40 \%$ of couples with infertility. The world's done studies that noticed increased morbidity of these children.

The aim of the study was to establish the structure of morbidity, as well as the frequency and types of congenital anomalies in children after IVF-a.

Prospective analysis include 102 infant born after IVF, in Gynecology - Obstetrics Clinic in Kragujevac.

In the period 2001-2009g birth incidence of children was $0.37 \%$. Children born at term $58.83 \%$. Apgar score above 7 had $74.51 \%, 25.49 \%$ were with different type of severe asphyxia while $2.94 \%$. (3) died. With Caesarean were terminated pregnancy in $73.68 \%$ cases. From 74 deliveries $8.82 \%$ was with pathological presentation. Overall morbidity was $83.78 \%$, early deliveries $(45.94 \%)$, asphyxia $(10.81 \%)$, intracranial hemorrhage $(14.80 \%)$. Congenital anomalies were diagnosed in $8.82 \%$ cases, including two syndromes L / Down and Dandy-Walker sy.

Infants born after IVF have a higher overall morbidity compared to other children. Incidence of gemellar pregnancy is high and twice more frequent pelvic presentation. Early birth was four times more, and a high percentage of intracranial hemorrhage. Congenital anomalies occur twice as often. Our conclusions can not be considered definitive because it requires a large number of children for the final positions.

Keywords: IVF, congenital anomalies, assisted fertilization 